This item was submitted to Loughborough's Research Repository by the author.

Items in Figshare are protected by copyright, with all rights reserved, unless otherwise indicated.

\title{
SABINE: A multi-purpose dataset of semantically-annotated social content
}

\section{PLEASE CITE THE PUBLISHED VERSION}

https://doi.org/10.1007/978-3-030-00668-6_5

\section{PUBLISHER}

(c) Springer Nature

\section{VERSION}

AM (Accepted Manuscript)

\section{PUBLISHER STATEMENT}

This work is made available according to the conditions of the Creative Commons Attribution-NonCommercialNoDerivatives 4.0 International (CC BY-NC-ND 4.0) licence. Full details of this licence are available at: https://creativecommons.org/licenses/by-nc-nd/4.0/

\section{LICENCE}

CC BY-NC-ND 4.0

\section{REPOSITORY RECORD}

Castano, Silvana, Alfio Ferrara, Enrico Gallinucci, Matteo Golfarelli, Stefano Montanelli, Lorenzo Mosca, Stefano Rizzi, and Cristian Vaccari. 2018. "SABINE: A Multi-purpose Dataset of Semantically-annotated Social Content”. Loughborough University. https://hdl.handle.net/2134/37086. 


\title{
SABINE: A Cross-Disciplinary Benchmark of Semantically-Annotated Social Content
}

\author{
Silvana Castano, Alfio Ferrara, Enrico Gallinucci, Matteo Golfarelli, Stefano Montanelli, Lorenzo Mosca, \\ Stefano Rizzi, and Cristian Vaccari
}

\begin{abstract}
Social Business Intelligence (SBI) is the discipline that combines corporate data with social content to let decision makers analyze the trends perceived from the environment. SBI poses research challenges in several areas, such as IR, data mining, and NLP; unfortunately, SBI research is often restrained by the lack of publicly-available, real-world data for experimenting approaches, and by the difficulties in determining a ground truth. To fill this gap we present SABINE, a modular benchmark in the domain of European politics. SABINE includes 6 millions bilingual clips crawled from 50000 web sources, each associated with metadata and sentiment scores; an ontology with 400 topics, their occurrences in the clips, and their mapping to DBpedia; two multidimensional cubes for analyzing and aggregating sentiment and semantic occurrences. We also propose a set of research challenges that can be addressed using SABINE; remarkably, the presence of an expert-validated ground truth ensures the possibility of testing approaches to the whole $\mathrm{SBI}$ process as well as to each single task.
\end{abstract}

Index Terms-Benchmark, social business intelligence, user-generated content.

\section{INTRODUCTION}

$\mathrm{D}$ URING the last decade, an enormous amount of usergenerated content (UGC) related to people's tastes, opinions, and actions has been made available thanks to the omnipresent diffusion of social networks and portable devices. This huge wealth of information has raised an intense interest from decision makers because it can give them a timely perception of the market mood and help them explain the phenomena of business and society. Social Business Intelligence (SBI) is the discipline that aims at combining corporate data with UGC to let decision makers analyze and improve their business based on the trends and moods perceived from the environment [1].

In the context of SBI, the most widely used category of UGC is the one coming in the form of textual clips. Clips can either be messages posted on social media or articles taken from on-line newspapers and magazines, or even customer comments collected on the corporate CRM. Digging information useful for decision makers out of textual UGC requires first crawling the web to extract the clips related to a subject area (e.g., politics), then enriching them in order to let as much information as possible emerge from the raw text. Enrichment activities may simply identify the

- S. Castano, A. Ferrara, and S. Montanelli are with DI, University of Milan, Italy.

E-mail: silvana.castano-alfio.ferrara-stefano.montanelli@unimi.it

- E. Gallinucci, M. Golfarelli, and S. Rizzi are with DISI, University of Bologna, Italy.

E-mail: enrico.gallinucci2—matteo.golfarelli-stefano.rizzi@unibo.it

- L. Mosca is with DFCS, University of Roma Tre, Italy.

E-mail: lorenzo.mosca@sns.it

- C. Vaccari is with Royal Holloway University of London, UK, and with University of Bologna, Italy.

E-mail: cristian.vaccari@rhul.ac.uk

Supported by the Italian Ministry of Education "Future in Research 2012" initiative (code RBFR12BKZH) for the project "Building Inclusive Societies and a Global Europe Online: Political Information and Participation on Social Media in Comparative Perspective" (www.webpoleu.net).

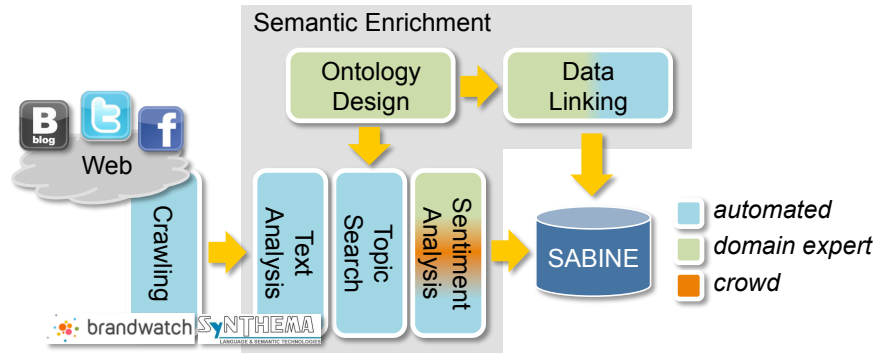

Fig. 1. The functional architecture of the SBI process which created SABINE

structured parts of a clip, such as its author, or even use NLP techniques to interpret each sentence, find the topics it mentions, and if possible assign a sentiment (i.e., positive, negative, or neutral) to it [2]. For instance, the tweet "UKIP's Essex county councillors stage protest against flying of EU flag at County Hall. Well done to them", in the subject area of EU politics, mentions topics "UKIP" and "protest" and has positive sentiment. Figure 1 sketches the overall SBI process.

From a scientific point of view, SBI stands at the crossroads of several areas in Computer Science such as Database Systems, Information Retrieval, Data Mining, Natural Language Processing, and Human-Computer Interaction. Though the ongoing research in these single fields has made available a bunch of results and enabling technologies for SBI, an overall view of the related problems and solutions is still missing. Besides, the peculiarities of SBI systems open new research problems in all the previous areas. On the other hand, research developments in SBI are often restrained by the lack of publicly-available, real-world data for experimenting and comparing approaches, and by the inherent difficulties in determining a ground truth for 


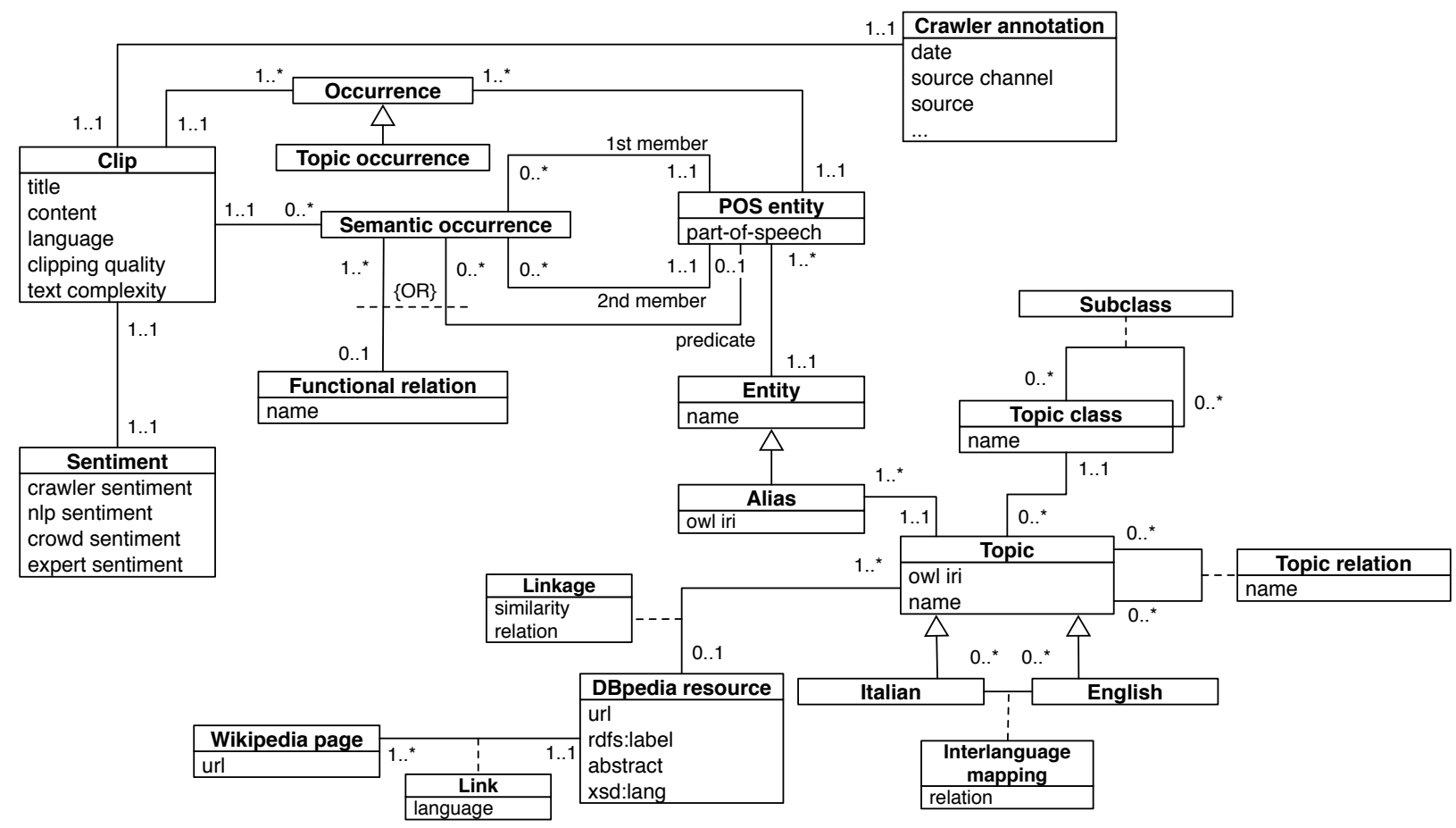

Fig. 2. UML model of SABINE

assessing the effectiveness of an approach.

To fill this gap, in this paper we present SABINE ( SociAl Business INtelligence bEnchmark), a modular benchmark in the domain of European politics with specific reference to the 2014 European elections. SABINE includes: 6 millions bilingual clips crawled from 50000 web sources, each one associated with metadata and sentiment scores; an ontology with 400 topics, their occurrences in the clips, and their mapping to DBpedia; and two multidimensional cubes for analyzing and aggregating sentiment and semantic occurrences for SBI analytics purposes. Remarkably, the presence of a manually-validated ground truth for each phase of the SBI process ensures the possibility of testing approaches to the whole process as well as to each single task. In this direction, our proposal is completed by a set of research challenges that can be addressed using SABINE; the task selection we propose is large and diverse enough to be sufficiently representative of a wide range of research tasks, ranging from content analysis, to semantic analysis, up to the more comprehensive SBI analytics.

The paper outline is as follows. In Section 2 we describe the benchmark content. In Section 3 we discuss the techniques adopted for building SABINE. In Section 4 we propose a set of SBI-related research tasks for SABINE. Finally, in Section 5 we draw the conclusions.

\section{The Content of SABine}

SABINE has been built as one of the results of the WebPolEU project (webpoleu.altervista.org), whose goal was to investigate the connection between politics and social media. SBI was used in the project as an enabling technology for analyzing the UGC generated in Italian and English during a timespan ranging from March, 2014 to May, 2014
TABLE 1

SABINE figures

\begin{tabular}{lll}
\hline Figure & ITA & ENG \\
\hline \# Web Sources & $25 \mathrm{~K}$ & $23 \mathrm{~K}$ \\
\# Topics & 434 & 409 \\
\# Topic Aliases & 364 & 300 \\
\# Entities & $1242 \mathrm{~K}$ & $2868 \mathrm{~K}$ \\
\# Clips & $2394 \mathrm{~K}$ & $3275 \mathrm{~K}$ \\
Avg Clip Length (\# Chars) & 1677 & 2026 \\
\# Entity Occurrences & $227 \mathrm{M}$ & $519 \mathrm{M}$ \\
\# Topic Occurrences & $14 \mathrm{M}$ & $23 \mathrm{M}$ \\
\# Semantic Occurrences & $35 \mathrm{M}$ & $48 \mathrm{M}$ \\
\hline
\end{tabular}

(the 2014 European Parliament Election was held on May 22-25, 2014). By analyzing digital literacy and online political participation, the research evaluated the inclusiveness, representativeness, and quality of the online political discussion.

The UML model of the SABINE content (except for the multidimensional part, whose content is described by Figure 4 is shown in Figure 2, while its quantitative features are summarized in Table 1 (see [3] for a more detailed profiling of the clips). The main content components of SABINE can be described as follows.

\subsection{Topics and Mappings}

SABINE provides about 400 relevant topics organized in a topic ontology built by domain experts (a team of five socio-political researchers). The topic ontology (modeled by classes Topic, Topic class, Subclass, and Topic relation in Figure 2) represents the set of concepts and relationships that, on the domain experts' judgement, are relevant to 


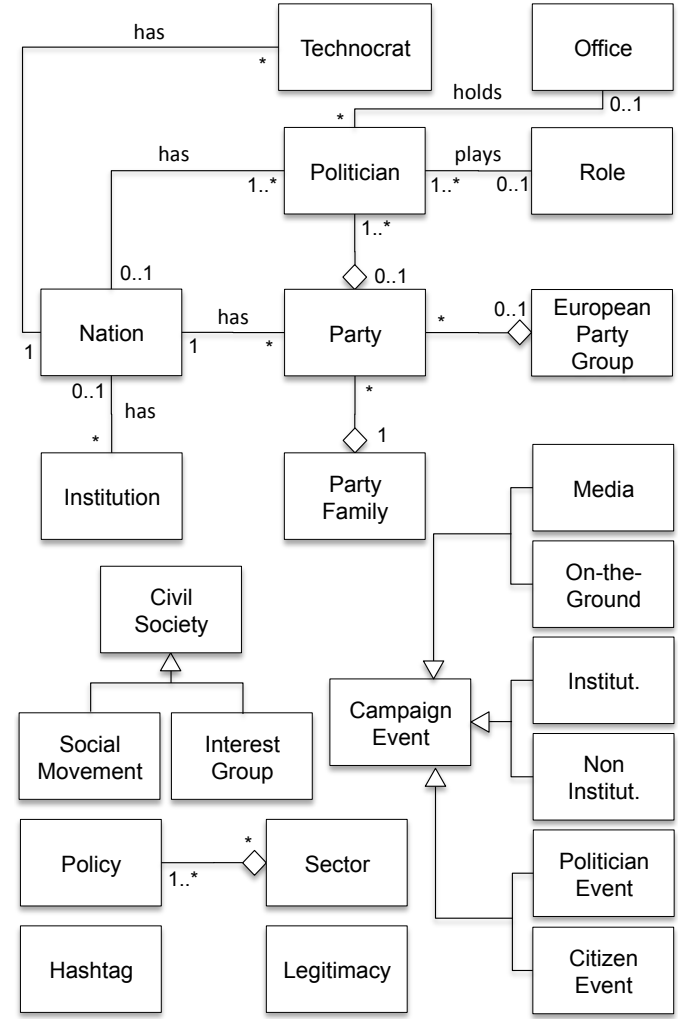

Fig. 3. The topic ontology represented as a UML class diagram

the subject area; its role in the SBI process is twofold: to act as a starting point for designing effective crawling queries on the one hand, and to support analyses based on relevant concepts (e.g., how often the public debt policy is mentioned) and on their aggregations (e.g., how often the sector of economics and its policies are discussed) on the other. The class diagram for the topic ontology of the sociopolitical subject area of SABINE benchmark is shown in Figure 3, for instance, topics "public debt" and "austerity" are instances of topic class Policy and are related to topic "economic policy" (Sector). To enable more accurate analyses, a large set of topic aliases (class Alias in Figure 2) has been identified and is available for topics (e.g., "tory" is an alias for "conservative").

Inter-Language mappings (class Interlanguage mapping) between corresponding topics in the two benchmark languages have been manually created by the domain experts. In most cases these mappings simply express an exact translation (e.g., "immigration" is mapped onto "immigrazione" with semantics owl:sameAs), whereas they are based on weaker semantic relationships when a concept is differently expressed in the two languages (e.g., "immigration" is mapped onto "migrante irregolare" — which means illegal migrant - with semantics sabine:related). A mapping has been found only in $86 \%$ of cases since, according to the experts' judgement, some topics are specific of either UK or Italy (e.g., "Scottish National Party" and "Quirinale"). Furthermore, topics have been linked to their corresponding DBpedia resources (classes DBpedia resource, Wikipedia page, Link, and Linkage). Linkage has been carried out automatically as described in Section 3.6 and then validated by domain experts.

\subsection{Clips and Annotations}

The benchmark provides a large corpus (around 6 millions) of raw clips (class Clip) extracted by the Brandwatch crawler from a broad set (almost 50 000) of web sources including social networks, blogs, and web sites. The most frequent clip sources are Facebook (53.8\% of the clips) and Twitter (27.5\% of the clips). The corpus is bilingual and comparable, i.e., it includes text in two languages (English and Italian) regarding similar topics [4], [5]. Each clip is associated with a set of metadata (class Crawler annotation); 40 attributes overall are provided, partly returned by the crawler (e.g., title, date, source MozRank, author information, and geolocalization) and partly manually annotated by the domain experts (e.g., source type).

Clips are enriched with other relevant information resulting from clip text analysis. In particular, each clip is associated with all its occurring entities and their parts-ofspeech or POSs (classes POS entity, Entity, and Occurrence). An entity is a concept that emerged from text analysis but is not necessarily a topic; parts-of-speech (POSs) are the roles taken by entities within a clip sentence (e.g., noun, verb, preposition). Among the set of entity occurrences, a relevant role is taken by the occurrences of topics and their aliases (class Topic occurrence). Finally, text analysis also led to the detection of more complex linguistic patterns involving multiple entities in the same sentence (classes Semantic occurrence and Functional relation). In particular, each semantic occurrence relates two entities (first and second member, respectively) by means of either a functional relation (e.g., agent or qualifier) or a predicate corresponding to an entity.

All clips are also annotated with two sentiment values (class Sentiment). The first one (crawler sentiment) is categorical (i.e., negative, neutral, positive); it has been determined for each clip by the Brandwatch crawler through rule-based techniques. The second one (NLP sentiment) is numerical; it has been determined by the SyN semantic engine for each clip sentence, then averaged for each clip (see Section 3.5. Finally, a subset of 2400 clips have also been labeled with a crowd sentiment, and half of these 2400 clips have further been labeled by domain experts (expert sentiment). This subset of manually-labelled clips has been created using a stratified sampling strategy based on the type of clip source (e.g., social network and blog) and on the clip sentiment.

Example 1. Here is an example of a SABINE clip: "Another compassionate conservative. Making fun of a parkinson's victim. Michael J Fox has more courage than you will ever hope to have". Some metadata for this clip are source $=$ "facebook.com", channel_type $=$ "facebook", source_type="Social network / Social media", country $=$ "US", and fb_role="audience". The only occurring topic is "conservative"; among the occurring entities we mention "compassionate", "victim", and "courage" (with POSs adjective, noun, and noun respectively). Text analysis led to find different semantic occurrences of entities with their POSs, for instance the one between "compassionate" and "conservative" (with POS adjective 
and noun, respectively, and functional relation qualifier) and the one between "have" and "courage" (with POS verb and noun, respectively, and functional relation $o b$ ject). The expert sentiment for this clip is -1 (i.e., negative), while both the crawler and the NLP sentiment are positive (because neither of the approaches was able to detect the irony in the sentence "Another compassionate conservative"). Another example of clip is "US President Barack Obama criticized Russia in a telephone call [...]", which shows a semantic occurrence between entities "Barack Obama" (POS proper noun) and "Russia" (POS proper noun) involving entity "criticize" as a predicate.

\subsection{Multidimensional Cubes}

These are ROLAP cubes providing an easy-to-query representation of the clip content and of the outcome of the clip enrichment process. The first cube, Sentiment, is centered on clips, and represents the set of topics appearing in each clip as well as the sentiment values computed for that clip. The second cube, Semantic Occurrence, is centered on the semantic occurrence of POS entities within clips and explicitly models couples of entities in the same sentence together with an optional predicate. The conceptual schemas of these cubes are depicted in Figure 4 using the DFM notation [6], where cube measures are listed inside the box, dimensions are circles directly attached to the box, and hierarchies are shown as DAGs of dimension levels. In particular, the hierarchy built on dimension Clip includes the crawler annotations, while the one built on Topic (called topic hierarchy from now on) derives from the topic ontology of Figure 3 and enables topic-based aggregations of clips in the OLAP front-end. For instance, a roll-up from Politician to Party and Party Family on the Sentiment cube allows to obtain the opinions about a wing as an average of the opinions about all the politicians belonging to the parties of that wing.

\section{SABine Construction Techniques}

To develop SABINE we followed the ad-hoc methodology described in [7], which has been conceived to support and speed up the initial design of an SBI process on the one hand and to maximize the effectiveness of the experts' analyses by continuously optimizing and refining all the process tasks on the other hand. Quick tuning iterations are probably the most distinctive feature of this type of projects, and are necessary to cope with the high fickleness of the topics covered in social conversations. In the following subsections we give further details on the techniques used for each task of the SBI process, using Figure 1 as a reference.

\subsection{Ontology Design}

Designing the topic ontology of the European politics subject area was mainly a methodological issue. Consistently with the methodology we followed [7], we initially carried out a macro-analysis to identify the themes relevant to the subject area (e.g., "culture") and a first set of topics (e.g., "school"). Then, the ontology design phase was specifically dedicated to collecting, for each theme, a comprehensive set of topics and to arrange them within an ontology (using
Protégé) by expressing inter-topic relationships. Along the whole project lifetime, the topic ontology was weekly tuned and refined (in collaboration with domain experts) to accommodate new topics, topic classes, and relationships; the final result is shown in Figure 3

As already mentioned, the topic hierarchy depicted in Figure 4 was derived from the topic ontology by applying standard techniques for multidimensional modeling of operational data sources, so as to enable topic-based aggregation of clips in an OLAP fashion.

\subsection{Crawling}

For keyword-based crawling we adopted the Brandwatch service (www.brandwatch.com), a commercial solution to ensure a satisfactory coverage of web sources during the project duration (three months). Brandwatch adopts a template-based engine, that is, it extracts only the informative UGC by detecting and discarding advertisements and banners (a process called clipping); it also drops duplicate clips using content aggregators.

The two main design activities related to crawling are source selection and crawling query design. As to source selection, Brandwatch already comes with its source base; however, our domain experts provided a set of about 100 additional domain-specific web sites (e.g., www. davidcameron.com) to be added to the source base and crawled. Similarly, a large number of non-relevant sources (e.g., www.tripadvisor.co.uk) had to be progressively eliminated from the source base because they were generating too many off-topic clips. Overall, in WebPolEU, dropping non-relevant sources and duplicate clips led to cut the number of collected clips from ten to six millions.

Keyword-based queries in Brandwatch rely on a set of 23 Boolean operators that allows to express filters on both textual features (e.g., the distance between two words) and metadata (e.g., the author's country and the web site name). We initially created a set of queries based on the topics in the domain ontology and on a few additional ones we discovered during source selection. To reduce the number of off-topic clips, a content relevance analysis was weekly performed by first asking the domain experts to manually label off-topic clips within a sample, then tuning the crawling queries to exclude those clips so as to increase the percentage of in-topic clips.

Example 2. As an example of how a query was transformed to exclude off-topic clips, we show the one aimed at retrieving the clips mentioning George Osborne (a conservative politician), which had to be changed from

$$
\text { (raw:"Osborne") OR “george osborne" }
$$

to

$$
\text { (raw:“Osborne” NOT (ozzie OR ozzy)) }
$$

$$
\text { OR "george osborne" }
$$

\subsection{Text Analysis}

For text analysis we used the SyN-Semantic Center (www. synthema.it) commercial engine. SyN was used for splitting clips in sentences and for extracting the single entities, 

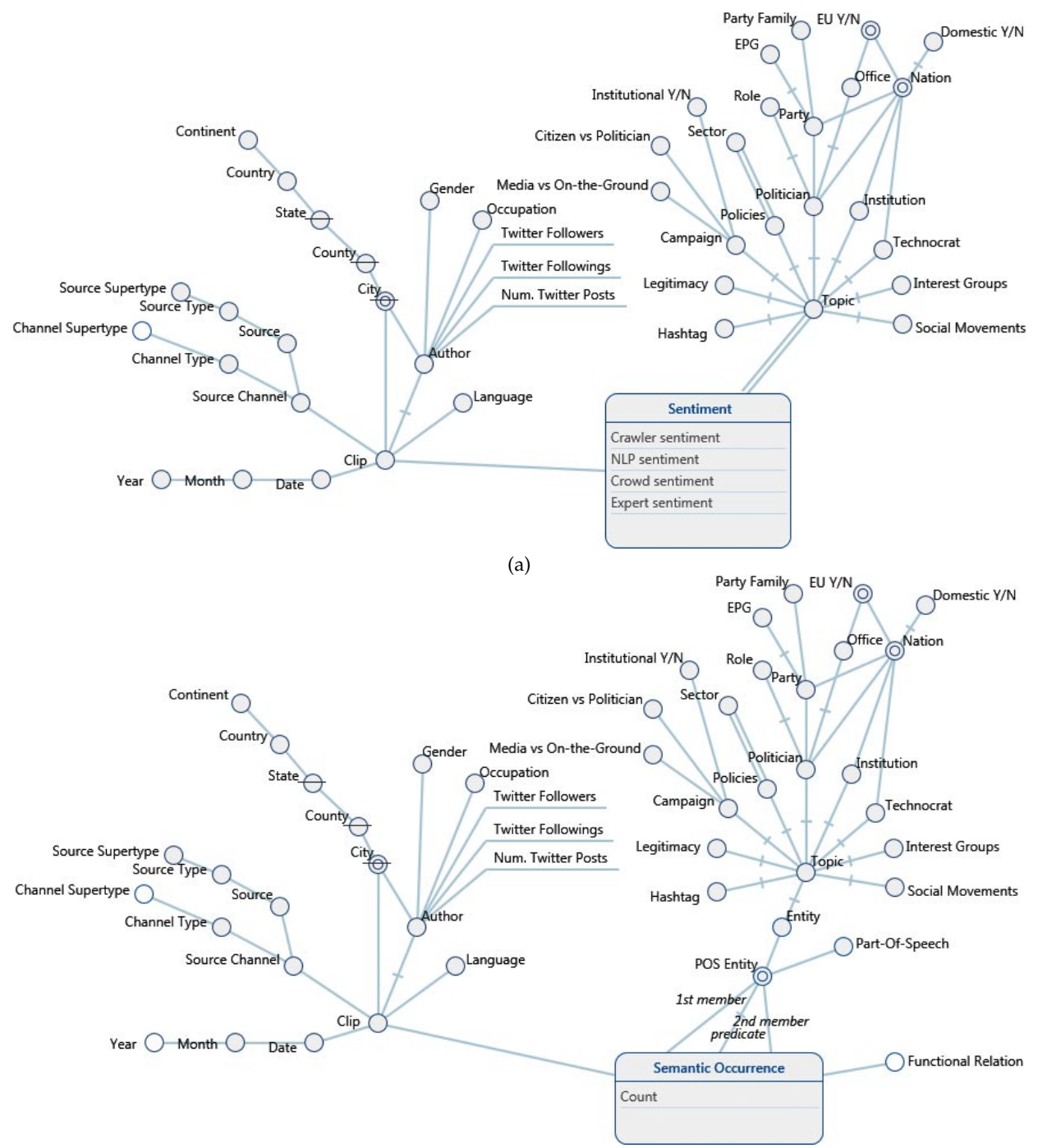

(b)

Fig. 4. A DFM representation of the Sentiment (a) and Semantic Occurrence (b) cubes (for drawing simplicity, some levels of the topic hierarchy are hidden)

their part-of-speech, and their semantic occurrences. The linguistic and semantic text analysis made by SyN is based on morpho-syntactic, semantic, semantic role, and statistical criteria. At the heart of the lexical system lies McCord's theory of slot grammars [8]. The system analyzes each sentence, cycling through all its possible constructions and trying to assign the context-appropriate meaning to each word by establishing its context. Each slot structure can be partially or fully instantiated and it can be filled with representations from one or more statements to incrementally build the meaning of a statement. The core of the system is the SyN ontology, developed through twenty years of experiences and projects.

\subsection{Topic Search}

With this term we refer to the task of indexing all the occurrences of a topic (or one of its aliases) within a clip. We relied on two different techniques for searching topic occurrences in SABINE. The first one is a simple text matching technique that retrieves the exact occurrences of topics and aliases, implemented in-house as a Java algorithm. The second one is based on the results of the text analysis made by SyN, which extracts all the occurring entities in a clip; in this case, topic occurrences are obtained by linking topics and aliases to the corresponding entities.

Clearly, both techniques have pros and cons. By avoiding all kinds of text analysis, the first technique typically trades a better performance in terms of speed with a lower accuracy of the results. In particular, the results tend to include the occurrences where a topic (or an alias) is used in the clip with different semantics from the one originally meant in the topic ontology. This problem arises when topics (or aliases) in the ontology are too generic.

Example 3. "Osborne" is an alias of topic "George Osborne". By identifying the exact matches of the word "Osborne", the first technique wrongly associates the occurrences of other people with the same surname (e.g., Peter Osborne, father of George) to the topic "George Osborne". Although this problem is similar to the one seen in Example 2 for crawling query design, it poses a different challenge (essentially because Peter Osborne frequently appears in in-topic clips). 
The second technique presents the opposite challenge: by carrying out an in-depth comprehension of the clip semantics, the entities produced by SyN tend to be very specific, possibly leading to the pulverization of the same concept into a wide set of entities. Therefore, this problem arises when topics (or aliases) in the ontology are intentionally generic.

Example 4. "university" is a topic of class Policy. The text analysis made by SyN produces a different entity for each specific university found in the clips (e.g., "Oxford University", "University of Cambridge", etc.). As a consequence, all these entities must then be manually associated with topic "university".

The adoption of both techniques enabled us to doublecheck the results and to track down the causes of conflicting results. Eventually, mismatches were manually solved in most cases, yielding a $91 \%$ agreement between the two techniques (over 14 millions occurrences).

\subsection{Sentiment Analysis}

Sentiment analysis is probably the hardest task in SBI; for this reason we included in SABINE both system-based and human-based sentiment scores. While system-based scores can be used as a baseline for testing other automatic techniques, human-based scores represent the ground truth.

- Crawler sentiment. This score, computed by Brandwatch, tags each clip of SABINE. The sentiment analysis component of Brandwatch is based on mining rules specifically developed for each language supported.

- NLP sentiment. SyN includes its own sentiment analysis component [9] whose score takes into account the negative or positive polarization of words and concepts, as well as the syntactical tree of the sentence being analyzed. SyN is sophisticated enough to modify the polarization of words based on the related adverbs, adjectives, conjunctions, or verbs, by taking in account specific functional-logic complements; it even tries to identify idiomatic or colloquial expressions and give an interpretation to negations. Each clip of SABINE is tagged with this score as well.

- Expert sentiment. This score was defined for a sample of 1200 clips (600 English + 600 Italian) by asking our domain experts to manually tag them. The clips are equally divided by media type and NLP sentiment (as computed by SyN). Besides defining the clip sentiment as either negative, neutral, or positive, the domain experts were also asked to rate, for each clip, its clipping quality (i.e., the amount of nonrelevant text present in the clip due to an inadequate template used by the crawler when clipping), which could impact on the difficulty of assigning the right sentiment, and its intrinsic text complexity (i.e., the effort of a human expert in assigning the sentiment due to irony, incorrect syntax, abbreviations, etc.).

- Crowd sentiment. This score was given to a sample of about 2400 clips $(1200+1200$, including the clips tagged by experts) through a crowdsourcing process. To this end, we selected a crowd of around 900 workers within a class of bachelordegree students in the field of humanities and political science at the University of Milano (average worker age is 21). Crowdsourcing activities were performed during one month and each worker tagged 46 clips on average. As a support we employed our Argo system (island.ricerca.di. unimi.it/projects/argo/, in Italian), which provides crowdsourcing functionalities based on multi-worker task assignment and consensus evaluation techniques [10]. In Argo, each clip to evaluate was represented as a choice task, meaning that each worker receiving a task to execute was asked to read a clip and select her preferred answer among three available options, namely positive, negative, and neutral sentiment. Each clip was assigned to a group of 6 different workers. A group member autonomously tagged each clip received and independently produced the answer according to her personal feeling and judgement. Each worker also had the opportunity to refuse a clip in case she recognized to have insufficient expertise for its sentiment evaluation. Given a clip, its sentiment score was defined as an answer agreement (i.e., consensus) among the members of the group that tagged that clip. Two workers agree on a clip when they assigned the same score to that clip. In Argo, consensus evaluation was enforced through a weightedvoting mechanism called supermajority, in which the answer of a worker has a weight corresponding to her reliability ${ }^{1}$ Supermajority was used to verify that the sentiment score $s$ having the highest degree of consensus within group $G$ was supported by a qualified majority larger than $50 \%$ [10]. In this case, score $s$ was assigned the corresponding clip. Conversely, when a qualified majority of workers was not found in $G$, the task was uncommitted and scheduled for re-execution by a different group of workers with higher reliability.

\subsection{Data Linking}

The goal of data linking is to link ontology topics to the Linked Data Cloud (linkeddata.org). In SABINE, this has been done by coupling automated techniques with manual validation and revision by domain experts; the single steps of the process are described in the following.

1. Topic aliases were used to retrieve a set of candidate DBpedia resources for each topic $t$ through the DBpedia Lookup Service (wiki.dbpedia.org/projects/ dbpedia-lookup).

2. The degree of similarity between $t$ and the retrieved candidates (if any) was evaluated through the HMatch matching algorithm [11]. HMatch takes into account both the linguistic information available for $t$ (i.e., its aliases) and the ontological information (i.e., the topic class of $t$ ).

1. The worker's reliability depends on the answers she provides to the assigned tasks; specifically, it is increased when the answer contributes to reach the consensus and it is decreased otherwise [10]. 
3. Topic $t$ was linked to the DBpedia resource $e$, among the candidates, yielding the highest degree of similarity. The link between $t$ and $e$ is formally defined as a 4-tuple of the form $\mathcal{L}_{t, e}=\left\langle t, e, \sigma_{t, e}, \rho_{t, e}\right\rangle$, where $\sigma_{t, e}$ is a real number in the range $[0,1]$ representing the degree of similarity between $t$ and $e$, and $\rho_{t, e}$ represents the semantics of the relation holding between $t$ and $e$.

4. Each resulting link $\mathcal{L}_{t, e}$ was submitted to domain experts to specify the most suitable semantics $\rho_{t, e}$, choosing among (i) owl:sameAs ( $t$ and $e$ have exactly the same meaning); (ii) sabine:narrower / sabine:broader (the meaning of $t$ is more specific/generic than the one of $e$ ); (iii) sabine:related (there is a positive association between the meanings of $t$ and $\epsilon^{2}$ If none of the previous options was deemed suitable by the domain experts, the link was marked as incorrect.

5. The links with semantics different from owl:sameAs and all the incorrect links were submitted to a second validation round, where domain experts manually found additional DBpedia resources to be associated with the corresponding topic with owl:sameAs semantics.

Example 5. As an example we propose the following link between topic "school" and DBpedia resource dbpedia:State_school:

\section{〈"school", dbpedia:State_school, 0.75, owl:sameAs〉}

In the first round of validation, experts confirmed that "school" can be linked to dbpedia:State_school, but with semantics sabine:broader (since school is broader than dbpedia:State_school). The resulting link was

\section{〈"school", dbpedia:State_school, 0.75, sabine:broader〉}

Since the semantics is different from owl:sameAs, the link was submitted to the second validation round, where we asked experts to manually find a DBpedia resource which actually has an owl:sameAs relation with "school". Experts found the DBpedia resource dbpedia:School, which leads to the addition of a second link for topic "school". The links resulting from the two validation rounds are then

$$
\begin{gathered}
\text { 〈"school", dbpedia:State_school, 0.75, sabine:broader〉 } \\
\text { 〈"school", dbpedia:School, 1.0, owl:sameAs〉 }
\end{gathered}
$$

In Table 2, we show some statistics about validation and refinement of English topics. Most of the automatically retrieved links have been considered correct with owl:sameAs semantics $(67 \%) ; 17 \%$ of the remaining links have been evaluated as correct but with semantics different from owl:sameAs. In particular, the automatic linking procedure tends to provide specific (rather than generic) DBpedia resources for topics. The automatic approach was incorrect in $16 \%$ of cases. We note also a positive correlation between the average degree of similarity associated with links and the positive evaluation provided by experts. This is important for associating a reliable degree of similarity to the links in

2. Note that we modeled relations similarly on SKOS (www.w3. org/TR/skos-reference/) concept relations. The reason why we redefined relation semantics rather than using SKOS is that topics and DBpedia resources are not defined as SKOS concepts.
TABLE 2

Results of the domain expert validation and revision for the English topics

\begin{tabular}{lll}
\hline Relation semantics & \# links & Avg. similarity \\
\hline owl:sameAs & 252 & 0.812 \\
sabine:narrower & 7 & 0.721 \\
sabine:broader & 39 & 0.756 \\
sabine:related & 17 & 0.781 \\
incorrect & 62 & 0.22 \\
\hline sub-total & 377 & \\
\hline expert-provided resource & 135 & 0.433 \\
\hline total & 512 & \\
\hline
\end{tabular}

the final benchmark. Finally, for 135 topics, domain experts provided a DBpedia resource as an owl:sameAs counterpart for the topic.

\section{Research TASKS}

In this section we describe the main research tasks that can be supported and evaluated using SABINE. Remarkably, to enable partial, ad-hoc downloads for each task, we subdivided SABINE into separate components shown as packages in Figure 5 A package models a component of the benchmark that can be downloaded separately; one package depends on another when an object of the former references an object of the latter. For each task, in Table 3 we summarize the SABINE component(s) to be taken in input, the expected task output, the ground truth we provide, and possible metrics for evaluating the task results with reference to the ground truth.

\subsection{Content Analysis Tasks}

Sentiment analysis. At the state of the art, the available techniques for sentiment analysis provide a satisfactory level of accuracy in narrow domains with limited dictionary and topics of discussion (e.g., movie reviews [2]). Finding an appropriate sentiment for a clip is still an open problem in wide domains and when sarcasm and irony are used -which is often the case for the clips in SABINE. The challenge we propose here is to assign a sentiment score (either positive, negative, or neutral) to each clip. As described in Section 3.5. SABINE provides four sentiment scores with different levels of certification. Noticeably, the 1200 clips tagged by domain experts have also been annotated with the level of difficulty (either normal or hard) the experts encountered in assigning the sentiment and with the presence/absence of undesired and irrelevant text due to crawler errors.

Topic search. Entity search in raw text is a classic information retrieval task. The approaches in this area typically uses lemmatization and stemming to increase their recall. Entity search turns to semantic search when additional information such as synonyms [12], ontologies [13], and context [14] are exploited. The research challenge we propose here is to find the occurrences of the given topics within the corpus of clips. The benchmark 


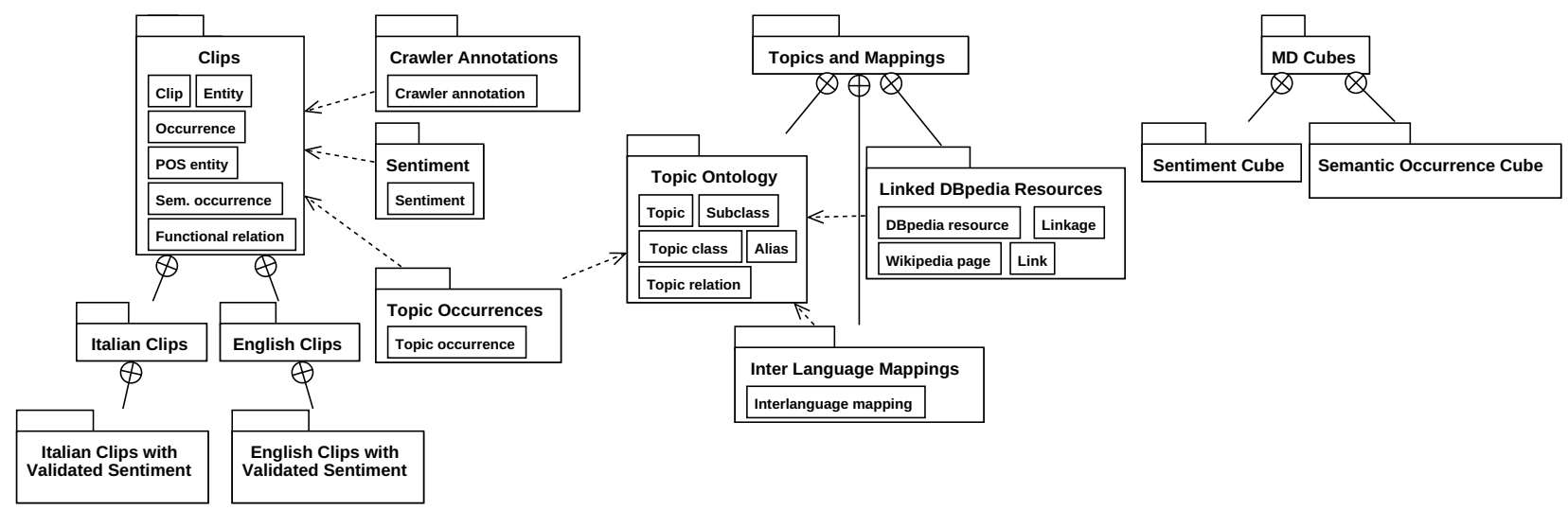

Fig. 5. Components of SABINE and their composition and dependency relationships

TABLE 3

Task overview

\begin{tabular}{|c|c|c|c|c|}
\hline Task & Input & Output & Ground truth & Evaluation \\
\hline \multicolumn{5}{|l|}{ Content Analysis Tasks } \\
\hline Sentiment analysis & Clips & Clip sentiment & Sentiment & Precision; Recall \\
\hline Topic search & Clips; Topic Ontology & Topic occurrences in clips & Topic Occurrences & $\begin{array}{l}\text { Precision; Recall; } \\
\text { Interpolated precision }\end{array}$ \\
\hline Document classification & Clips & Classes of clips & Crawler Annotations & $\begin{array}{l}\text { Purity; Mutual informa- } \\
\text { tion; Rank index }\end{array}$ \\
\hline \multicolumn{5}{|l|}{ Semantic Analysis Tasks } \\
\hline Cross-language analysis & Topic ontology; Clips & $\begin{array}{l}\text { Matches between English and } \\
\text { Italian topics }\end{array}$ & Inter-Language Mappings & Precision; Recall \\
\hline Topic discovery & Clips & Topics & Topic Ontology & Precision; Recall \\
\hline Data linking & Topic Ontology & $\begin{array}{l}\text { Links between topics and } \\
\text { DBpedia resources }\end{array}$ & Linked DBPedia Resources & $\begin{array}{l}\text { Precision; Recall; } \\
\text { Interpolated precision }\end{array}$ \\
\hline \multicolumn{5}{|l|}{ SBI Analytics Tasks } \\
\hline $\begin{array}{l}\text { Multidimensional } \\
\text { modeling }\end{array}$ & $\begin{array}{l}\text { Topic Occurrences; Sentiment; Clips; } \\
\text { Crawler Annotations; Topic Ontology }\end{array}$ & Multidimensional cubes & MD cubes & Sum squared error \\
\hline SBI analytics & Clips & Query answers & MD Cubes & Sum squared error \\
\hline
\end{tabular}

can be used to check the efficiency and effectiveness of original techniques. As to effectiveness, the ground truth provided by SABINE consists of the occurrences of the topics in the clips as obtained through two different and independent techniques (see Section 3.4). Both traditional and semantic search techniques can be applied since (i) topic aliases provide a set of synonyms for topics, and (ii) the topic ontology provides the complete list of topics and their relationships (e.g., a Sector includes several Policies; a Politician belongs to a Party).

Document classification. Document classification aims at associating a document with one or more document classes. There is a wide literature on this subject and a variety of different approaches have been proposed, including for example probabilistic techniques such as Naive Bayes models [15], techniques based on the vector space model of documents and on support vector machine [16], and techniques based on matrix decomposition [17|. Document classification may lead to different results according to different perspectives, especially when classification is driven by subjective criteria - as in the case of sentiment analysis. Consequently, for document classification we propose to rely on the crawler annotations, i.e., metadata associated to the documents according to objective criteria (see Sec- tion 2). In particular, annotations include the dimensions of language, author, source, and date. For author, source, and date, metadata also provide further levels of aggregation. Each of these dimensions can be used to group clips in different classes with the desired level of aggregation, from relatively small and specific classes to large and generic ones. Examples of clip classes along the date dimensions are those grouping clips by date of publication (specific) and those grouping clips by year (generic). According to the classification scheme derived from the crawler annotations, it is then possible to evaluate a classification approach either with a supervised strategy, where classes are known apriori, or with an unsupervised strategy, starting from the clips only. The goal in both cases is to rebuild the same classification that can be derived from crawler annotations.

\subsection{Semantic Analysis Tasks}

Cross-language analysis. This area has several relevant applications. For example, cross-language information retrieval addresses the problem of finding information in one language in response to queries expressed in another, while cross-language text categorization uses labeled documents in one language to classify documents in another language. A requirement shared by these applications is the availability of a bilingual dictionary. Traditional approaches assume 
that such dictionaries are either given a priori (and typically obtained through a time-consuming manual effort) or automatically acquired from parallel corpora [18]. Since parallel corpora are still a scarce resource in several languages and contexts, recent researches employ comparable corpora [4], [5] and unsupervised object matching methods [19], [20]. Both methods are promising in terms of language portability because they do not require external language resource. The research challenge we propose in SABINE is to automatically derive the best match between inter-language topics. The input is the sets of topics in the two languages as well as the set of clips representing comparable corpora. The benchmark includes the correct matches, which have been manually defined by the domain experts.

Topic discovery. Several contributions have been provided in the area of topic discovery, mainly in the research field of topic modeling starting from Latent Semantic Analysis (LSA) approaches [21] back in the 90's to Latent Dirichlet Allocation (LDA) approaches 22 in the 2000's. Besides latent analysis, other important contributions are based on clustering techniques [23], [24]. More recently, combinations of LDA, mainly based on Gibbs sampling, and clustering have been proposed to handle topic modeling with very large text collections [25]. Topic modeling is an important reference in the social sciences domain and it has been applied to several research issues, including the impact and dissemination of research [26], social media [27], and the study of changes in technology \& innovation [28|. Judging about the presence of a topic in a document is a highly subjective and domain-dependent task. Thus, evaluating automatic solutions requires a gold standard of high quality, where topics are validated by domain experts. We propose to exploit our topic ontology and topic occurrences in clips, which were both validated by domain experts, in order to evaluate topic discovery strategies and tools. In particular, we envisage both supervised strategies, where the number and categories of topics are given a-priori, and non-supervised strategies, where topics are discovered from the clips only and then the topics discovered are compared with those validated by our experts. The evaluation may be based on the set of topics that are discovered in the corpus, without considering which topic occurs in which clip, or be extended to assess also the topic occurrences in clips.

Data linking. In general terms, data linking is the task of determining whether two object descriptions can be linked one to the other to represent the fact that they refer to the same real-world object in a given domain or the fact that some kind of relation holds between them [29]. Usually, this task is performed based on the evaluation of the degree of similarity among different data instances describing real-world objects across heterogeneous data sources, under the assumption that the higher the similarity between two data descriptions, the higher the probability that the two descriptions actually refer to the same object. In our challenge, we propose to link a subset of the topics with their corresponding DBpedia resources. The ground truth we provide has been manually validated by domain experts for both English and Italian. In the field of data linking, a clear and widely accepted definition of the meaning of links between data is still missing. In general, the results of data linking is a set of links associated with same-as semantics and these links are represented using (or abusing) the owl:sameAs OWL relation. However, in the literature there is a wide spectrum of different techniques, capable of discovering different kinds of possible links between object descriptions, ranging from weak correspondences to strong relations of identity. Aimed at supporting validation also for approaches capable of discovering the specific semantics of data links, we provide not only same-as links between topics and DBpedia resources, but also a set of links with other semantics, including analogy, generic similarity, specialization, and generalization (see Section 3.6). The evaluation of automatic techniques for data linking can be performed either by verifying if the correct DBpedia resource has been associated with a topic, disregarding the semantics of the association, or by taking into account also the semantics of the link discovered and checking if it is compatible with the links provided by domain experts.

\subsection{SBI Analytics Tasks}

Multidimensional modeling. The goal here is to design a set of multidimensional cubes that summarize and store the knowledge generated by semantic enrichment, in terms of both efficiency and effectiveness. Possible design solutions range from traditional star schemata to more sophisticated approaches like meta-stars [30] or those proposed in [31]. The cubes included in the MD Cubes component can than be used as a baseline for comparison.

SBI Analytics. The research tasks proposed so far can be considered technical tasks aimed at enriching raw clips and enable SBI analyses. To complete the benchmark we also propose a functional task, namely, finding the answers to a set of enquiries proposed by our domain experts:

- Q1: Which is the most discussed sector in relationship with each political party?

- Q2: Which are the most discussed topics for each source type?

- Q3: Are there any topics whose volume of discussion significantly changes from UK to Italy? Note that this is a cross-language enquiry and requires to find out those topics whose number of occurrences are most unbalanced between Italian and English clips.

- Q4: How does the sentiment about each politician and technocrat change along time?

The ground truth we provide for this task are the answers obtained by directly querying our multidimensional cubes. The domain experts verified that these answers are fully compatible with the real social and political phenomena they directly observed during the election period, thus certifying the validity of the ground truth. Remarkably, this task enables an end-to-end assessment of a whole SBI process starting from clips, possibly enriched with different combinations of the benchmark components.

\section{Conclusion}

In this paper we have presented SABINE, a benchmark of semantically annotated social content in the domain of 
European politics. SABINE aims to constitute a publiclyavailable, real-world data benchmark for experimenting and comparing the most commonly performed SBI tasks, crossing the various involved disciplines ranging from Database Systems, Information Retrieval, Data Mining, up to Natural Language Processing and Human-Computer Interaction. SABINE has been designed and properly packaged for modular download to enable the evaluation of a wide variety of research tasks, either separately or in combination, ranging from those more focused on content analysis, to those related to semantic analysis up to more comprehensive SBI analytics, by ensuring that interesting and technical challenges of the tasks will emerge from benchmark implementation.

A main technical advance of SABINE is the availability of multiple, complementary, and validated enrichments of the social content (i.e., textual clips) in form of metadata, annotations, sentiment scores, and DBpedia mappings. The availability of a user-validated ground truth, either by domain experts or by crowdsourcing or both, for each enrichment phase represents a further technical advance of SABINE, to target the purpose of providing a comprehensive and effective benchmark environment.

To download SABINE: SABINE is available for download at big.csr.unibo.it/?q=sabine: packages are made available as compressed archive files containing JSON files (the Clips package), OWL files (the Topics and Mappings package and all its sub-packages), and CSV files (all other packages).

\section{REFERENCES}

[1] E. Gallinucci, M. Golfarelli, and S. Rizzi, "Advanced topic modeling for social business intelligence," Information Systems, vol. 53, pp. 87-106, 2015.

[2] B. Liu and L. Zhang, "A survey of opinion mining and sentiment analysis," in Mining Text Data. Springer, 2012, pp. 415-463.

[3] M. Francia, E. Gallinucci, M. Golfarelli, and S. Rizzi, "Social business intelligence in action," in CAISE, to appear, 2016.

[4] C. Chu, T. Nakazawa, and S. Kurohashi, "Iterative bilingual lexicon extraction from comparable corpora with topical and contextual knowledge," in Computational Linguistics and Intelligent Text Processing. Springer-Verlag, 2014, pp. 296-309.

[5] P. G. Otero, "Learning bilingual lexicons from comparable English and Spanish corpora," MT Summit xI, pp. 191-198, 2007.

[6] M. Golfarelli and S. Rizzi, Data Warehouse design: Modern principles and methodologies. McGraw-Hill, 2009.

[7] M. Francia, M. Golfarelli, and S. Rizzi, "A methodology for social BI," in IDEAS, 2014, pp. 207-216.

[8] M. C. McCord, Slot grammar. Springer, 1990.

[9] F. Neri, C. Aliprandi, F. Capeci, M. Cuadros, and T. By, "Sentiment analysis on social media," in ASONAM, 2012, pp. 919-926.

[10] S. Castano, A. Ferrara, L. Genta, and S. Montanelli, "Combining crowd consensus and user trustworthiness for managing collective tasks," Future Generation Computer Systems, vol. 54, 2016.

[11] S. Castano, A. Ferrara, and S. Montanelli, "Matching ontologies in open networked systems: Techniques and applications," JoDS, vol. V, 2005.

[12] C. Carpineto and G. Romano, "A survey of automatic query expansion in information retrieval," ACM Computing Surveys, vol. 44, no. 1, p. 1, 2012.

[13] M. Fernández, I. Cantador, V. López, D. Vallet, P. Castells, and E. Motta, "Semantically enhanced information retrieval: An ontology-based approach," Web Semantics: Science, Services and Agents on the World Wide Web, vol. 9, no. 4, pp. 434-452, 2011.

[14] J. Pound, P. Mika, and H. Zaragoza, "Ad-hoc object retrieval in the web of data," in WWW, 2010, pp. 771-780.
[15] L. H. Lee and D. Isa, "Automatically computed document dependent weighting factor facility for naïve Bayes classification," Expert Syst. Appl., vol. 37, no. 12, pp. 8471-8478, 2010.

[16] L. H. Lee, C. H. Wan, R. Rajkumar, and D. Isa, "An enhanced support vector machine classification framework by using Euclidean distance function for text document categorization," Applied Intelligence, vol. 37, no. 1, pp. 80-99, 2012.

[17] K. Maruta, H. Nagai, and T. Nakamura, "Document classification with varied viewpoints using matrix decomposition," in IIAI-AAI, 2015, pp. 154-159.

[18] P. Fung, "A statistical view on bilingual lexicon extraction: from parallel corpora to non-parallel corpora," in Machine Translation and the Information Soup. Springer, 1998, pp. 1-17.

[19] T. Hirao, T. Iwata, and M. Nagata, "Latent semantic matching: Application to cross-language text categorization without alignment information," in ACL (2), 2013, pp. 212-216.

[20] N. Quadrianto, A. J. Smola, L. Song, and T. Tuytelaars, "Kernelized sorting," IEEE PAMI, vol. 32, no. 10, pp. 1809-1821, 2010.

[21] T. K. Landauer, P. W. Foltz, and D. Laham, "An introduction to latent semantic analysis," Discourse processes, vol. 25, no. 2-3, pp. 259-284, 1998.

[22] D. M. Blei, A. Y. Ng, and M. I. Jordan, "Latent Dirichlet allocation," Journal of Machine Learning Research, vol. 3, pp. 993-1022, 2003.

[23] Y.-B. Liu, J.-R. Cai, J. Yin, and A. W.-C. Fu, "Clustering text data streams," Journal of computer science and technology, vol. 23, no. 1, pp. 112-128, 2008.

[24] X. Zhou, X. Hu, X. Zhang, X. Lin, and I.-Y. Song, "Contextsensitive semantic smoothing for the language modeling approach to genomic IR," in SIGIR, 2006, pp. 170-177.

[25] N. Nagwani, "Summarizing large text collection using topic modeling and clustering based on mapreduce framework," Journal of Big Data, vol. 2, no. 1, pp. 1-18, 2015.

[26] Y. Dong, R. A. Johnson, and N. V. Chawla, "Will this paper increase your h-index?: Scientific impact prediction," in Int. Conf. on Web Search and Data Mining, 2015, pp. 149-158.

[27] P. Bogdanov, M. Busch, J. Moehlis, A. K. Singh, and B. K. Szymanski, "Modeling individual topic-specific behavior and influence backbone networks in social media," Social Network Analysis and Mining, vol. 4, no. 1, pp. 1-16, 2014.

[28] Y. Zhang, G. Zhang, H. Chen, A. L. Porter, D. Zhu, and J. Lu, "Topic analysis and forecasting for science, technology and innovation: Methodology with a case study focusing on big data research," Technological Forecasting and Social Change, vol. 105, pp. 179-191, 2016.

[29] A. Ferrara, A. Nikolov, and F. Scharffe, "Data linking for the semantic web," Semantic Web: Ontology and Knowledge Base Enabled Tools, Services, and Applications, vol. 169, 2013.

[30] E. Gallinucci, M. Golfarelli, and S. Rizzi, "Advanced topic modeling for social business intelligence," Information Systems, vol. 53, pp. 87-106, 2015.

[31] U. Dayal, C. Gupta, M. Castellanos, S. Wang, and M. GarcíaSolaco, "Of cubes, DAGs and hierarchical correlations: A novel conceptual model for analyzing social media data," in ER, 2012, pp. $30-49$.

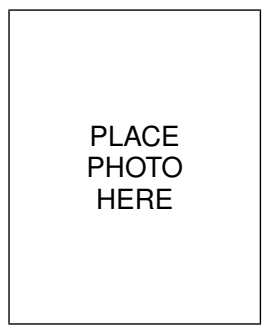

